\title{
Analysis of the Effect of Different Treatment Schemes on Helicobacter Pylori Infection on Improving the Quality of Life of Patients
}

\section{Zhi Zong}

Hospital of Shandong Jianzhu University, Jinan 250101, China
Abstract: Objective: To analyze the effect of different treatment schemes on the quality of life of patients with Helicobacter pylori infection. Methods: From June 2019 to November 2020, 96 patients with Helicobacter pylori infection were selected and randomly assigned Combined treatment with omeprazole, amoxicillin and clarithromycin was named group A; Group B was treated with omeprazole, amoxicillin, clarithromycin and colloidal bismuth pectin; Group $\mathrm{C}$ was given omeprazole, amoxicillin, clarithromycin and metronidazole. Results: The symptom score and gastroscope score of group $\mathrm{B}$ or $\mathrm{C}$ were lower than those of group A $(P<0.05)$; The incidence of adverse reactions and HP eradication rate in group $C$ were lower than those in group $\mathrm{B}$, and those in group B were lower than those in group $\mathrm{A}(P<0.05)$; The quality of life score of group $\mathrm{C}$ was higher than that of group B, and that of group B was higher than that of group A, $P<0.05$. Conclusions: The sequential therapy of omeprazole, amoxicillin, clarithromycin and metronidazole in patients with Helicobacter pylori infection can improve the HP eradication rate, improve the quality of life of patients, which is safe and efficient.

Keywords: Helicobacter pylori infection; Different treatment schemes; Quality of life score

Publication date: January, 2021

Publication online: 31 January, 2021

"Corresponding author: Zhi Zong, 453094800@ qq.com

The incidence rate of Helicobacter pylori infection is high, and has a certain infection rate. It is related to many factors such as social economy, population density and public health. After Helicobacter pylori infection, the typical symptoms of patients are heartburn, acid regurgitation, stomachache and halitosis, and with the progress of the disease, it can turn into gastritis or gastric cancer, and even endanger life and health. At this time, abdominal discomfort, acid regurgitation, belching, dull pain and other symptoms can appear, and the disease is not cured repeatedly, leading to the decline of the quality of life of patients ${ }^{[1]}$. In clinical practice, anti infective drugs are mostly used in patients with Helicobacter pylori infection. However, due to the frequent use of antibiotics and the increase of drug resistance, the traditional triple therapy has been unable to meet the needs of patients. In order to further improve the clearance rate of Helicobacter pylori, the clinical trial of bismuth containing quadruple therapy and sequential therapy ${ }^{[2]}$. This paper aims to analyze the application value of different treatment schemes in patients with Helicobacter pylori infection, taking 96 patients with Helicobacter pylori as samples to carry out the research, the report is as follows.

\section{Material and methods}

\subsection{Information}

The study was conducted in June 2019 and terminated in November 2020. A total of 96 cases were diagnosed as Helicobacter pylori infection and randomly divided into two groups. In group A, there were 17 males and 15 females, the youngest and oldest were 21 and 68 years old respectively, with an 
average age of ( $44.17 \pm 3.48)$ years, and the average course of disease was $(3.94 \pm 1.21)$ years; In group $\mathrm{B}$, there were 18 males and 14 females, the youngest and oldest were 22 and 69 years old respectively, with an average age of $(44.35 \pm 3.47)$ years, and the average course of disease was $(4.03 \pm 1.19)$ years; In group $\mathrm{C}$, there were 19 males and 13 females, the youngest and oldest were 23 and 70 years old respectively, with an average of $(44.76 \pm 3.51)$ years old, and the average course of disease was (4.11 \pm 1.21) years. All samples were selected for carbon 14 breath test, the results showed that HP was positive, gastroscopy was accompanied by gastritis, gastric ulcer or duodenal ulcer, and they all volunteered to participate. The patients who were treated with bismuth, antibiotics or proton pump inhibitors before admission, gastric surgery history, drug allergy, kidney and heart dysfunction were excluded ${ }^{[3]}$. This study was approved by the ethics committee. The data of 96 patients with Helicobacter pylori infection were compared, $P>0.05$.

\subsection{Treatment}

Group A was treated with traditional triple therapy, omeprazole (produced by Shandong Luoxin Pharmaceutical Co., Ltd.), twice a day, 40mg each time; Amoxicillin (produced by Zhuhai federal Pharmaceutical Co., Ltd.) was given twice a day, $1.0 \mathrm{~g}$ each time; Clarithromycin (produced by Zhejiang Beide Pharmaceutical Co., Ltd.) was given twice a day, with a single dose of $0.5 \mathrm{~g}$. The treatment lasted for 10 days.

Group B was given omeprazole, 40mg twice a day; Amoxicillin was given twice a day with a single dose of $1.0 \mathrm{~g}$; Clarithromycin was given twice a day, $0.5 \mathrm{~g}$ each time; Colloidal bismuth pectin (produced by Shanxi Zhendong ante biopharmaceutical Co., Ltd.) was given once a day, $150 \mathrm{mg}$ each time.The treatment lasted for 10 days.
Group C was treated with sequential therapy. Five days before administration, omeprazole and amoxicillin were given twice a day, with a single dose of $40 \mathrm{mg}$ and $1.0 \mathrm{~g}$ respectively; Five days after administration, clarithromycin and metronidazole (produced by Kangmei Pharmaceutical Co., Ltd.) were given twice a day, with a single dose of $0.5 \mathrm{~g}$ and $0.4 \mathrm{~g}$, respectively, for a total of 10 days.

\subsection{Efficacy evaluation}

After drug intervention, the symptoms of patients with Helicobacter pylori infection disappeared, no erosion, edema and hyperemia were found under gastroscope, and the ulcer developed to scar stage; It is effective if the symptoms are improved, the symptoms of erosion, edema and hyperemia are relieved, and the ulcer area disappears more than $50 \%$; If the symptoms still exist, the erosion, edema and hyperemia under gastroscope are not improved, and the ulcer area is reduced less than $50 \%$, it is invalid $^{[4]}$.

\subsection{Statistical study}

SPSS 33.0 was used to calculate the data of patients with Helicobacter pylori infection. The changes of each index during the treatment period were recorded in the form of $\%,(\bar{x} \pm s)$. The differences between groups were verified by $\chi^{2}$ and $\mathrm{t}$ forms. There was significant difference between the two groups $(P<$ $0.05)$.

\section{Results}

\subsection{Comparison of symptom score and gastro- scopy score between groups}

The symptom score and gastroscope score of group $\mathrm{B}$ and group $\mathrm{C}$ were lower than those of group $\mathrm{A}$ after different administration regimens, $P<0.05$. See Table 1. 
Table 1. Difference analysis of symptom score and gastroscope score (score, $\bar{x} \pm s$ )

\begin{tabular}{ccccc}
\hline Integral index & time & Group A & Group B & Group C \\
\hline Symptom score & Before treatment & $1.81 \pm 0.35$ & $1.82 \pm 0.36$ & $1.79 \pm 0.33$ \\
& After treatment & $1.42 \pm 0.11$ & $0.85 \pm 0.07$ & $0.76 \pm 0.05$ \\
Gastroscope integral & Before treatment & $1.61 \pm 0.29$ & $1.58 \pm 0.26$ & $1.59 \pm 0.27$ \\
& After treatment & $1.04 \pm 0.15$ & $0.68 \pm 0.17$ & $0.51 \pm 0.11$ \\
\hline
\end{tabular}

2.2 Compare the difference of adverse reactions and HP eradication rate

lower than those in group B, and those in group B

After different treatment, the incidence of adverse were lower than those in group A, $P<0.05$. As shown reactions and $\mathrm{HP}$ eradication rate in group $\mathrm{C}$ were in Table 2.

Table 2. Difference analysis of adverse reactions and HP eradication rate $(n, \%)$

\begin{tabular}{cccc}
\hline Index & Group A & Group B & Group C \\
\hline HP eradication rate & $21(65.63)$ & $26(81.25)$ & $31(96.88)$ \\
Headache & $8(25.00)$ & $4(3.13)$ & $1(3.13)$ \\
Diarrhea & $9(28.13)$ & $5(15.63)$ & $2(6.25)$ \\
Constipation & $7(21.88)$ & $3(93.75)$ & $0(0.00)$ \\
Skin Itch & $5(15.63)$ & $2(6.25)$ & $0(0.00)$ \\
Abdominal discomfort & $6(18.75)$ & $3(93.75)$ & $0(0.00)$ \\
\hline
\end{tabular}

2.3 Comparison of quality of life scores between groups

After the occurrence of Helicobacter pylori infection, taking different treatment regimens, the quality of life scores of group $\mathrm{C}$ were higher than those of group B, the quality of life scores of group B were higher than those of group A, $P<0.05$. As shown in Table 3 .

Table 3. Quality of life score analysis table (points, $\bar{x} \pm s$ )

\begin{tabular}{cccc}
\hline Index & Group A & Group B & Group C \\
\hline Physiological function & $76.18 \pm 2.36$ & $83.27 \pm 2.98$ & $94.28 \pm 3.25$ \\
Social function & $75.49 \pm 2.41$ & $84.32 \pm 2.76$ & $93.76 \pm 3.18$ \\
Mental health & $75.87 \pm 2.34$ & $85.66 \pm 2.59$ & $94.31 \pm 3.36$ \\
Emotional function & $76.24 \pm 2.29$ & $84.97 \pm 2.61$ & $93.82 \pm 3.41$ \\
Somatic pain & $77.31 \pm 2.15$ & $84.81 \pm 2.58$ & $94.55 \pm 3.59$ \\
\hline
\end{tabular}

\section{Discussion}

Helicobacter pylori infection is closely related to a variety of gastric diseases, so the clinical treatment is still to eradicate HP infection.Combined with the analysis of clinical practice, HP is highly sensitive to drugs, but after human infection with HP, it mostly exists under the gastric mucous membrane and on the surface of epithelial cells, so oral administration is affected by the gastric mucous layer barrier, the effect is poor, and during the long-term drug intervention, the body drug resistance increases, which is not conducive to the prognosis of patients ${ }^{[5]}$. In recent years, the treatment of HP has been a hot spot in clinical research. The commonly used drugs include antibiotics, proton pump inhibitors, nitroimidazole and bismuth agents. The commonly used administration regimens are triple therapy, quadruple therapy and sequential therapy. However, with the increase of the abuse rate of antibiotics and the drug resistance of Helicobacter pylori patients, the eradication rate of traditional triple therapy is limited, so people try to use colloidal bismuth pectin on the basis of triple therapy ${ }^{[6]}$. Colloidal bismuth pectin is a new type of drug, which belongs to colloidal bismuth preparation. Its biological macromolecular pectin can replace other small molecular bismuth acid preparations, with high colloidal properties, which can increase the protection of gastric mucosa, enhance the clearance rate of HP, and promote the deposition of HP cell wall ${ }^{[7]}$. In addition, colloidal bismuth pectin can also promote bubble formation, accelerate cell wall rupture, inhibit the activity of bacterial enzymes, and then reduce the metabolic infection of bacterial enzymes. However, relevant scholars believe that during bismuth preparation treatment, the risk of complications such as headache, 
skin allergy and melena is high, which can affect the prognosis, so the clinical application is still controversial ${ }^{[8]}$. Combined with the analysis of the research results, group a received conventional triple therapy, group B received colloidal bismuth pectin quadruple therapy, and group $\mathrm{C}$ received sequential therapy; The incidence of adverse reactions and HP eradication rate in group $\mathrm{C}$ were lower than those in group B, and those in group B were lower than those in group $\mathrm{A}$; The quality of life score of group $\mathrm{C}$ was higher than that of group $\mathrm{B}$, and that of group $\mathrm{B}$ was higher than that of group A, $P<0.05$.It is suggested that the sequential treatment of omeprazole, amoxicillin, clarithromycin and metronidazole can effectively improve the clinical symptoms, eradicate $\mathrm{HP}$, and improve the quality of life of patients.

In conclusion, the traditional triple therapy and metronidazole sequential therapy in patients with Helicobacter pylori infection can significantly improve the HP eradication rate and reduce the risk of adverse reactions, which can be widely used in clinical practice.

\section{References}

[1] Huang Q. Analysis of the efficacy of the four-combination regimen containing amoxicillin and clavulanate potassium for gastric Helicobacter pylori infection [J]. Chinese Journal of Pharmacy, 2019(1): 15.
[2] Liu HJ. Effect of integrated traditional Chinese and western medicine on gastric mucosa in patients with helicobacter pylori infection gastric ulcer [J]. Chinese Journal of Medicine, 2019(9): 110 .

[3] Li MH. Comparative analysis of therapeutic efficacy of different therapeutic schemes in the treatment of Helicobacter pylori infection with peptic ulcer in children [J].World Latest Medical Information Abstracts, 2018, 18(96): 335-336.

[4] Xiong KP. Eradication and safety of three drug schemes in the treatment of active chronic gastritis R with Helicobacter pylori infection [J]. Health for All (Middle Edition), 2018, 12(1): 144-145.

[5] Zhong B. Clinical efficacy of different drug regimens on chronic gastritis patients with Helicobacter pylori infection and its effect on HP clearance [J]. Infectious Pharmacy, 2019, v.16; No. 86 (9) : 66-69.

[6] Fang M, Huang T H. Economic effect of different drug therapy on the eradication of Helicobacter pylori [J]. Shenzhen Journal of Integrated Traditional Chinese and Western Medicine, 2019, v.29;No. 246 (17): 178-179.

[7] Guo S L, Gao CC. Analysis of different drug regimens in the treatment of chronic gastritis caused by Helicobacter pylori infection [J]. Health Frontiers, 2018, 027(005):160.

[8] Luo SH, Liu WB, Xie HY. Comparative analysis of clinical efficacy of different drug regimens in the treatment of Helicobacter pylori (Hp) infected gastric ulcer [J].Clinical Research of Traditional Chinese Medicine, 2018, 10(36): 6365 . 